\title{
HOVERING STABILITY OF HELICOPTERS WITH ELASTIC CONSTRAINTS
}

\author{
Paul E. I. Pounds* \\ Department of Mechanical Engineering \\ Yale University \\ New Haven, Connecticut 06511 \\ Email: paul.pounds@yale.edu
}

\author{
Aaron Dollar \\ Department of Mechanical Engineering \\ Yale University \\ New Haven, Connecticut 06511 \\ Email: aaron.dollar@yale.edu
}

\section{ABSTRACT}

Aerial vehicles are difficult to stabilize, especially when acted upon by external forces. A hovering vehicle in contact with objects and surfaces must maintain flight stability while subject to forces imparted to the airframe through the point of contact. These forces couple with the motion of the aircraft to produce distinctly different dynamics from free flight. While external contact is generally avoided, extending aerial robot functionality to include contact with the environment during flight opens up new and useful areas such as perching, object grasping and manipulation. In this paper, we present a general elastic contact constraint model and analyze helicopter stability in the presence of those contacts. As an example, we evaluate the stability of a proof-of-concept helicopter system for manipulating objects using a compliant gripper that can be modeled as an elastic linkage with angular reaction forces. An off-the-shelf PID flight controller is used to stabilize the helicopter in free flight, as well as during the aerial manipulation task. We show that the planar dynamics of the object-helicopter system in vertical, horizontal and pitch motion around equilibrium are shown to remain stable, within a range of contact stiffnesses, under unmodified PID control.

NOMENCLATURE

$\begin{array}{ll}a_{n}, b_{n}, \text { etc. } & \text { Routh-Hurwitz array elements. } \\ a & \text { Blade lift slope. } \\ c_{R D} & \text { Rotor damping constant. } \\ C & \text { Flight controller transfer function. } \\ d & \text { Center of gravity-linkage offset. } \\ G & \text { Translational dynamics poles. } \\ F_{x}, F_{z} & \text { Elastic linkage reaction forces. } \\ F_{G E} & \text { Ground effect force. }\end{array}$

*Address all correspondence to this author.
$F_{R D} \quad$ Vertical rotor damping force.

$g \quad$ Acceleration due to gravity.

$H, H^{\prime} \quad$ Open-loop plant pitch-input transfer functions.

$H_{c l} \quad$ Closed-loop plant pitch-input transfer functions.

$h \quad$ Rotor height above center of gravity.

I Helicopter pitch inertia.

$k \quad$ PID flight controller system gain.

$k_{d} \quad$ PID flight controller derivative gain.

$k_{i} \quad$ PID flight controller integral gain.

$k_{G E} \quad$ Ground effect spring constant.

$k_{x}, k_{x}^{\prime} \quad$ Linkage horizontal spring constants.

$k_{z}, k_{z}^{\prime} \quad$ Linkage vertical spring constants.

$k_{\theta}, k_{\theta}^{\prime} \quad$ Linkage pitch spring constants.

$l$

$m, m_{0}$

$q_{1}$

$q_{2}$

$R$

$T_{F A}, T_{G E}$

$u$

$x$

Z

$\beta$

$\theta$

$\rho$

$\sigma$

$\tau \quad$ Elastic linkage torsional moment.

$\omega \quad$ Rotor angular velocity.

\section{INTRODUCTION}

Unmanned Aerial Vehicles (UAVs) are becoming increasingly important in a variety of fields [1]. However, UAVs 'look, but don't touch', with minimal interaction with their environment, and significant effort has gone into preventing UAVs from 


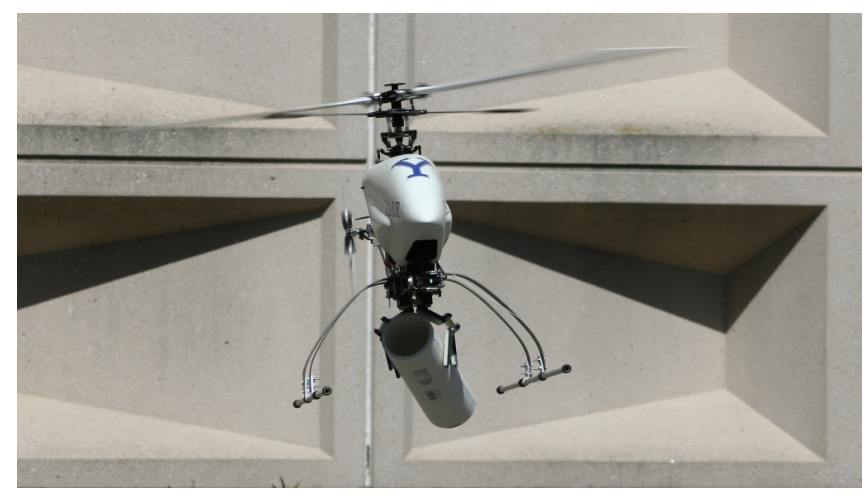

Figure 1. AERIAL MANIPULATION TESTBED CARRYING A TUBE.

contacting objects around them. It is desirable to extend the scope of UAV functionality to include contacting, picking-up and manipulating objects. The flying ability of UAVs promises capabilities not available to any wheeled or legged terrestrial robot: rapid traversal of impassible terrain, movement around 3D environments and an unlimited vertical workspace. Hovering UAVs such as helicopters and quadrotors are especially appealing platforms for object interaction: they can come to a stop over an object in order to interact with it, whereas a fixed-wing aircraft must maintain a minimum velocity.

During contact with the environment, stability of the aircraft in flight must be guaranteed [2]. In this paper, we present a general contact model and examine the dynamic stability of planar helicopters with elastic constraints. Conventional external loads applied to helicopters, such as tether loads and landing gear oleo, apply unidirectional forces - either vertical compression or tension. However, generalized contact forces are applied bi-directionally - these loading conditions create structurally different dynamic behavior. Aircraft interacting with objects and surfaces require novel frameworks for analyzing flight stability; this model is applicable to extended cases of conventional aircraft loading, as well as to new cases such as aircraft perching and object grasping.

Of specific interest is the possibility of manipulating objects while hovering, without the need to land. This minimizes the time and energy needed to perform a manipulation task and permits the robot to acquire objects from terrain not suitable for landing, such as at the tops of power lines, radio masts or on the surface of water. The contact constraints imposed by this task are challenging, and may serve to destabilize the craft if not properly understood and accounted for. In addition to presenting a general contact model and stability analysis, we examine the specific case of grasping external objects from a helicopter platform with a compliant gripper mounted ventrally under the airframe.

\section{Aerial Manipulation}

The difficulties of aerial manipulation are numerous. The vehicle must maintain hover position of the helicopter over the target object accurately enough for capture with an end effector

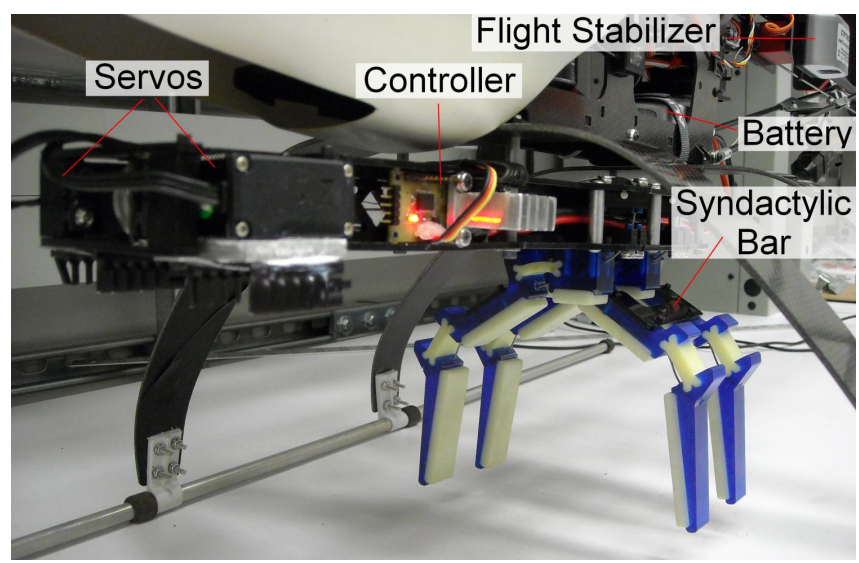

Figure 2. COMPLIANT GRIPPER MODULE.

such as a gripper or hook, and reject disturbances and biases from aerodynamic effects. Earlier efforts to overcome the imprecision of hovering vehicles have relied heavily on structuring of the target object to simplify the task, for example using magnets $[3,4]$ and hoops [5]. However, this greatly limits the variety of objects the robot can grasp.

The approach taken in this work is to use a compliant underactuated manipulator, based on the SDM Hand [6], mounted ventrally between the skids of a $4.3 \mathrm{~kg}, 1.5 \mathrm{~m}$ rotor, T-Rex 600 ESP radio control helicopter (Fig. 1). The gripper consists of four fingers with two elastic joints each, actuated by a parallel tendon mechanism that balances loads across each digit; it has a grasp span of $115 \mathrm{~mm}$ (Fig. 2). The helicopter is stabilized with a Helicommand flight controller, directed by a human pilot. The aircraft and gripper can carry loads above $1 \mathrm{~kg}$.

The special characteristics of the hand design - open-loop adaptive grasping, wide finger span, insensitivity to positional error [6] - closely match the challenges associated with the UAV manipulation task, allowing for a very simple, light-weight mechanism, without the need for imposed structural constraints on the load. To acquire an object, the helicopter approaches the target, descends vertically to hover over the target and then closes its gripper. Once a solid grasp is achieved, the helicopter ascends with the object.

\section{Flight Stability and External Contact}

Contacting and grasping objects while flying raises questions of continued aircraft flight stability [2]. When a robot helicopter with an elastic gripper has hold of an object, but has not yet applied enough thrust to ascend with it, contact forces will be transmitted through the gripper to the airframe. It is possible that these added dynamics will not be correctly compensated-for by the flight controller and thus destabilize the helicopter, leading to a crash. Throughout all modes of operation, the vehicle must guarantee flight stability to remain in the air.

Automatic flight controllers for small-scale helicopters are now commercially available. Use of off-the-shelf avionics is beneficial as it keep costs and overhead down and reduces development time. However, these flight controllers may not be 
adaptable to deal with the additional forces transmitted through the compliant gripper. It is desirable to show that a standard control architecture, in this case Proportional Integral Derivative (PID) control, will remain stable during external contact for a given gripper and helicopter configuration.

A compliant gripper may be approximated as a linkage (conceptualized as a compressible tether) connecting the immovable object and the helicopter. Research into tethered unmanned helicopter stability has been conducted since the 1960s. An early paper describes two fundamental flight modes of tethered helicopters [7]: stability of attitude due to the low connection point of the tether, and pure instability in position, the so-called 'pendulum' mode. These dynamics have been exploited to produce a stable unmanned rotor platform that flew at the end of its tether in a local equilibrium where the tether tension, weight and rotor thrust were balanced by automatic control [8]. More recently, efforts have focussed on autonomous landing of helicopters on ships in rough weather using a tether winch $[9,10]$. All of these papers consider the helicopter to be flying far from the tether point, where the line tension and direction is approximately constant.

In this case, where the elastic link is short, the aircraft will be operating exclusively around the equilibrium directly above the tether point, in low-velocity flight. The applied load cannot be treated as a constant either in magnitude or direction, nor always in tension, and consequently the mechanics are quite distinct from previous models. A different analytical approach must be taken which specifically includes the unique dynamics loadings transmitted to the airframe through the gripper.

\section{Roadmap}

In this paper we present a general force model and dynamic stability analysis of a planar helicopter with elastic contact constraints. We examine the specific case of grasping external objects from a helicopter platform with a compliant gripper mounted ventrally under the airframe. A 3-DOF helicopter flight model, including vertical rotor motion damping and ground effect, is presented, along with a PID controller that stabilizes the pitch dynamics in flight.

The combined helicopter-linkage system is analyzed for stability using a bogie suspension approximation of the SDM hand grasping a fixed object. The bogie suspension is simplified to a single pin-jointed prismatic link with torsional springs fitted at each end. The Routh-Hurwitz criterion is used to show that that the vertical motion of the system is always stable, and that the coupled pitch-longitudinal motion is conditionally stable for a subset of linkage stiffness configurations.

Finally, we assess the parameters of the proof-of-concept helicopter UAV fitted with the compliant gripper, and conclude that it will be stable during hovering object capture.

\section{HELICOPTER FLIGHT MODEL}

Much work has been done to control autonomous rotorcraft flight attitude, and the dynamics of helicopters in hover are well

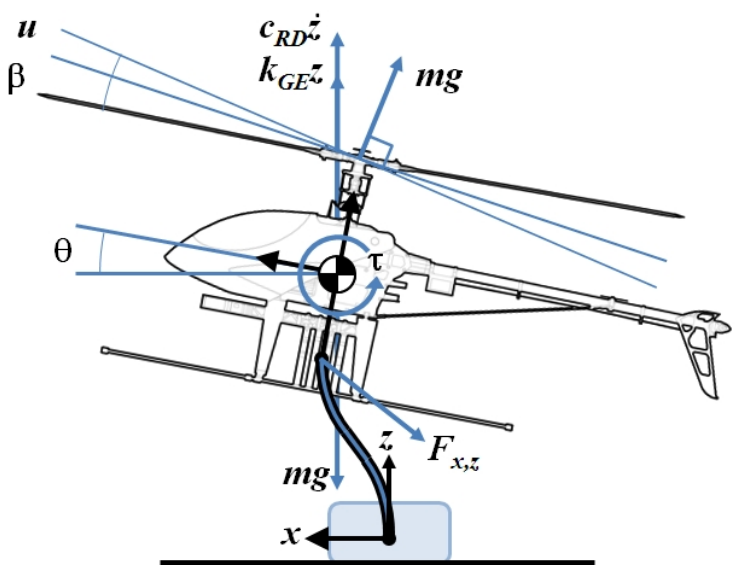

Figure 3. HELICOPTER DYNAMIC MODEL WITH ELASTIC LINKAGE.

understood $[3,11,12]$. Due to the largely decoupled lateral and longitudinal dynamics of helicopters around hover, a simple, linear, planar model is useful for analyzing the stability of both the free-air and constrained systems. In this paper, longitudinal dynamics are considered, but the analysis is equally applicable to lateral flight near hover.

The rigid-body dynamics of the linearized planar helicopter in hover are ${ }^{1}$ :

$$
\begin{aligned}
m \ddot{x} & =-m g \beta-m g \theta-m g u+F_{x} \\
m \ddot{z} & =-F_{R D}-F_{G E}+F_{z} \\
I \ddot{\theta} & =m g h \beta+m g h u+\tau
\end{aligned}
$$

where $m$ is the mass of the helicopter, $\mathrm{I}$ is the pitch rotational inertia of the helicopter, $g$ is acceleration due to gravity, $x, z$ and $\theta$ are the longitudinal, vertical and angular position of the center of mass, $h$ is the rotor height above the center of mass, $\beta$ is the first harmonic longitudinal rotor flapping angle, $u$ is the cyclic pitch control input, $F_{R D}$ is rotor inflow damping, $F_{G E}$ is effective ground effect force, and $F_{x}, F_{z}$ and $\tau$ are the longitudinal force, vertical force and pitch moment applied by the linkage.

We will consider a helicopter with a rotor that is free to 'flap' (or pivot) at the center like a see-saw. In horizontal motion, the on-coming wind causes an imbalance in lift between the blades on either side of the rotor disc. This causes the rotor plane to pitch upward, changing the angle of attack of each blade until a new equilibrium is reached.

The angled rotor directs some of its thrust aft, slowing the helicopter and producing a pitching moment. Flapping dynamics are a crucial part of helicopter stability analysis, even at low speeds. The rotor pitch response is extremely fast, and so it can be represented analytically, without need for additional states.

At low speeds, the flapping angle, $\beta$, produced by a zero flapping hinge-offset rotor head is an approximately linear combination of the longitudinal translation and pitch velocities:

\footnotetext{
${ }^{1}$ Rotor thrust is taken as constant, exactly canceling helicopter weight, and so is not included in the vertical dynamics.
} 


$$
\beta=q_{1} \dot{x}-q_{2} \dot{\theta}
$$

where $q_{1}$ and $q_{2}$ are constant parameters of the rotor [12].

In free air, rotor wake disperses beneath the helicopter and attenuates some distance downstream. However, when close to the ground (within approximately one rotor-diameter distance), the wake of the rotor is contained by the surface underneath it, creating a cushion of air referred to as 'ground effect'. This cushioning increases the lift generated by the rotor and is experienced by the pilot as a repulsive force resisting the helicopter's descent.

The ratio of thrust in ground effect, $T_{G E}$, to thrust in free air, $T_{F A}$, is [13]:

$$
\frac{T_{G E}}{T_{F A}}=\frac{16 z^{2}}{16 z^{2}-R^{2}}
$$

The increase in thrust close to ground is treated as force applied as a function of distance away from the trimmed equilibrium hover altitude, $z_{0}$ (taken as $z=0$ ). This can be treated like a spring force $F_{G E}=k_{G E} z$, where:

$$
k_{G E}=\left[\frac{32 z_{0}}{R^{2}-16 z_{0}^{2}}+\frac{512 z_{0}^{3}}{\left(R^{2}-16 z_{0}^{2}\right)^{2}}\right]
$$

Vertical motion of a rotor through its own induced flow changes the local flow angle of attack at blades, which alters the amount of lift produced. This change in thrust is in the opposing direction of motion, producing vertical damping. The damping force produced is $F_{R D}=c_{R D} \dot{z}$ [12], where:

$$
c_{R D}=\left[\frac{a}{4} \frac{\sigma}{\omega R}\right] \rho \pi R^{2}(\omega R)^{2}
$$

\section{Flight Control and Free-Air Stability}

Helicopter pitch and longitudinal motion are strongly interdependent, but vertical motion is effectively decoupled from these around hover. Solving the longitudinal translation-pitch equations together produces a single-input-single-output transfer function between the cyclic control input and the pitch angle in free flight:

$$
H=\frac{m g h G-m^{2} g^{2} h q_{1}}{\mathrm{I} s^{2} G+m g h q_{2} G s-m^{2} g^{2} h q_{1}\left(q_{2} s-1\right)}
$$

where $G=m s+m g q_{1}$.

Helicopter flight dynamics are intrinsically unstable and require feedback control from onboard sensors for autonomous hover. Commercial flight controllers using PID are now readily available for small-scale helicopters. These systems are affordable and straight-forward to use but lack the flexibility of tailor-made avionics. They cannot easily be adapted to dynamics distinctly different from expected helicopter behavior.

The transfer function for a PID controller has the form:

$$
C=k\left(1+\frac{k_{i}}{s}+k_{d} s\right)
$$

where $k$ is the control gain, and $k_{i}$ and $k_{d}$ are the integral and differential control parameters.
The stability of the controlled system can be assessed by examining the characteristic polynomial of the closed loop transfer function, $H_{c l}$ :

$$
H_{c l}=\frac{C H}{1+C H}
$$

The polynomial is the sum of the products of the numerators $(n)$ and denominators $(d)$ of $C$ and $H$ :

$$
C_{n} H_{n}+C_{d} H_{d}
$$

Substituting (8) and (9), this becomes:

$$
s^{3}+\left(\frac{m g h}{\mathrm{I}}\left(q_{2}+k k_{d}\right)+q_{1} g\right) s^{2}+k \frac{m g h}{\mathrm{I}} s+\frac{m g h}{\mathrm{I}}\left(k k_{i}+q_{1}\right)
$$

As the controlled helicopter is stable in free air, this polynomial is known to be stable and its coefficients satisfy the the RouthHurwitz criterion. The criterion states that for a dynamical system to be stable, its characteristic polynomial must have all positive coefficients, and that leading entries in the Routh-Hurwitz array derived from those coefficients must be positive. In the case of a third order polynomial [14]:

$$
s^{3}+a_{1} s^{2}+a_{2} s+a_{3}
$$

the lead elements of the array are given by:

$$
\begin{aligned}
& b_{1}=\left(a_{1} a_{2}-a_{3}\right) / a_{1} \\
& c_{1}=a_{3}
\end{aligned}
$$

Given (12) and (14) it can be shown that for free-air stability:

$$
-\mathrm{I} q_{1} g-\mathrm{I} k k_{i}+\mathrm{I} q_{1} g k+m g h k\left(q_{2}+k k_{d}\right)>0
$$

which is later used in factorizing polynomial elements for the tethered case.

\section{CONTACT MODEL}

In the specific application of grasping from a helicopter, the elastic linkage being investigated is a compliant gripper mechanism. The gripper consists of four fingers, each with two links and two elastic flexures at the proximal and distal joints, with stiffnesses $k_{1}$ and $k_{2}$ respectively. In the planar case, the gripper is treated as two axis-symmetric non-slip contact points representing opposing pairs of fingers (see Fig. 4$)^{2}$. The rotor thrust balances the weight of the helicopter; it cannot lift the grasped object and so the object is considered rigidly fixed to the ground.

Each finger can be modeled as a prismatic spring with an torsional spring at the proximal joint, where the equivalent spring stiffnesses, $k_{r}$ and $k_{\theta}$, are given by:

\footnotetext{
${ }^{2}$ The lateral view of the gripper is shown here for clarity - the same model can be used for both lateral and longitudinal motion of the gripper, where $k_{1}$ is the out-of-plane stiffness of the proximal flexural element.
} 


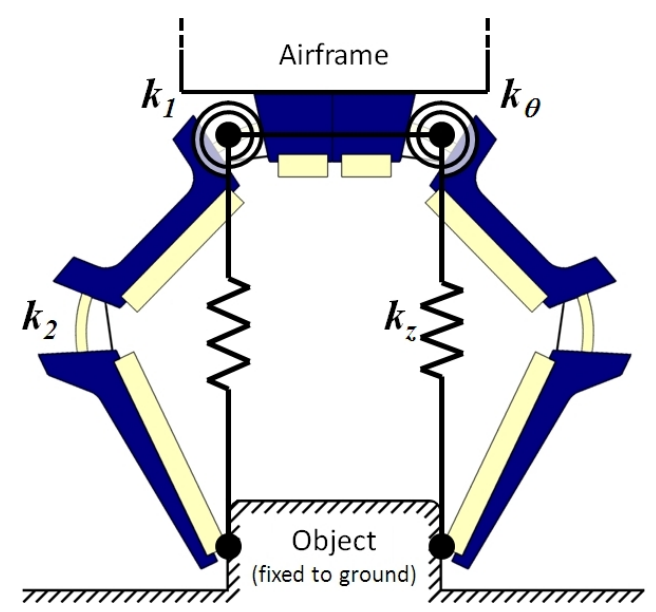

Figure 4. BOGIE SUSPENSION GRIPPER MODEL.
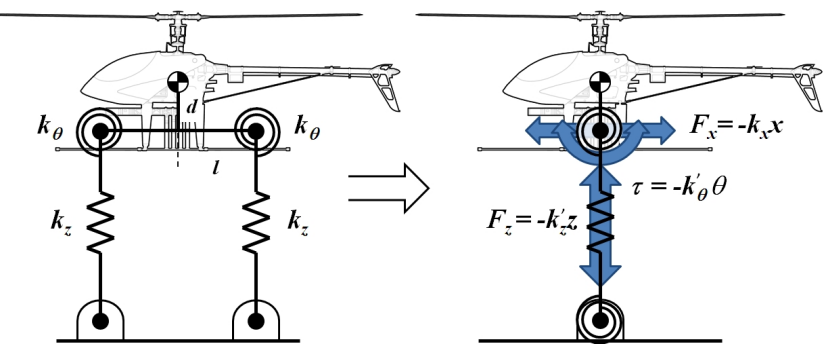

Figure 5. BOGIE SUSPENSION SINGLE LINK APPROXIMATION.

$$
\begin{aligned}
& k_{\theta}=k_{1} \\
& k_{z}=\frac{k_{1}+2 k_{2}}{3}
\end{aligned}
$$

where $k_{\theta}$ is the torsional spring stiffness and $k_{z}$ is the prismatic spring stiffness, assuming that the proximal and distal links are of equal length.

Around equilibrium, the elastic forces and torques applied due to $x, z$ and $\theta$ motion are approximately decoupled. Thus, the planar bogie suspension configuration can be approximated as a single elastic linkage with tension springs at both pin joints (see Fig. 5). The springs of the equivalent single linkage, $k_{z}^{\prime}, k_{\theta}^{\prime}$ and $k_{x}$, are computed by:

$$
\begin{aligned}
& k_{z}^{\prime}=2 k_{z} \\
& k_{\theta}^{\prime}=2 k_{z} l^{2}+2 k_{\theta} \\
& k_{x}=2 k_{\theta}
\end{aligned}
$$

where $l$ is the distance between the tether point and the bogie pin joint - corresponding to the proximal joint of the gripper.

This model is valid for the case where the helicopter thrust exactly equals its own weight; lateral stiffness induced by vertical thrust is zero in this case. However, as the helicopter begins to apply thrust to ascend, the vertical force applied to the end of the linkage will induce a lateral kinematic stiffness in addition to the elastic stiffness of the linkage:

$$
F_{x}=-\left(k_{x}+\frac{\delta T}{d}\right) x
$$

where $\delta T$ is the additional applied thrust, up to the weight of the carried object $m_{0} g$. The combined stiffness term is denoted as $k_{x}^{\prime}$. For large carried masses, this stiffness can be greater than that of the linkage.

When the thrust applied exceeds the combined weight of the helicopter, the object leaves the ground and the craft transitions to suspended load behavior, the mechanics of which have been explored in detail elsewhere $[15,16]$. This linkage model can also be extended to other related constraint scenarios such as motion of flexible landing skids on landing or adapted to novel cases such as compliant connections to objects suspended by swarms of UAVs.

\section{VERTICAL STABILITY}

Motion in the $\mathrm{Z}$ direction is independent of longitudinal and pitch motion, and is not directly regulated by the flight controller. Consequently, it must be intrinsically stable to reject disturbances.

Using the standard linear spring-displacement model, the equation of motion of the system in vertical motion becomes:

$$
m \ddot{z}=-c_{R D} \dot{z}-\left(k_{G E}+k_{z}^{\prime}\right) z
$$

The Laplace transform yields the characteristic polynomial:

$$
s^{2}+\frac{c_{R D}}{m} s+\frac{k_{G E}+k_{z}^{\prime}}{m}
$$

The coefficients of this characteristic polynomial are always positive. The Routh-Hurwitz criterion provides the following condition for stability:

$$
\frac{k_{G E}+k_{z}^{\prime}}{m}>0
$$

As these terms are physical parameters greater than zero, this condition is always true and the system is stable in $z$.

\section{PITCH-TRANSLATION STABILITY}

The horizontal dynamics of the system can be analyzed in the same way as the vertical dynamics. Note that the longitudinal translation of the system is coupled back into the pitch dynamics through the moment produced around linkage offset $d$. The dynamic equation of the tethered plant is:

$$
H^{\prime}=\frac{m g h G-m g q_{1}\left(m g h s-d k_{x}^{\prime}\right)}{\mathrm{I} G s^{2}+m g h q_{2} G s-m g q_{1}\left(m g h s-d k_{x}^{\prime}\right)\left(q_{2} s-1\right)+G k_{\theta}^{\prime}}
$$

where

$$
G=m s^{2}+m g q_{1} s+k_{x}^{\prime}
$$

gives the poles of the translational dynamics. 
Using the control law given in (9), the characteristic equation of the closed-loop system is the fifth-order polynomial:

$$
s^{5}+a_{1} s^{4}+a_{2} s^{3}+a_{3} s^{2}+a_{4} s+a_{5}
$$

where

$$
\begin{aligned}
a_{1}= & \left(\mathrm{I} q_{1} m g+m^{2} g h\left(q_{2}+k k_{d}\right)\right) /(\mathrm{I} m) \\
a_{2}= & \left(k m^{2} g h+m k_{\theta}^{\prime}+\mathrm{I} k_{x}^{\prime}\right) /(\mathrm{I} m) \\
a_{3}= & m g\left(k k_{d}\left(h+q_{1} d\right) k_{x}^{\prime}+m h k k_{i}\right. \\
& \left.+q_{1}\left(k_{\theta}^{\prime}+q_{2} d k_{x}^{\prime}+m g h\right)+q_{2} h k_{x}^{\prime}\right) /(\mathrm{I} m) \\
a_{4}= & k_{x}^{\prime}\left(k_{\theta}^{\prime}+m g\left(k h+k q_{1} d-q_{1} d\right)\right) /(\mathrm{I} m) \\
a_{5}= & k_{x}^{\prime} k k_{i} m g\left(h+q_{1} d\right) /(\mathrm{I} m)
\end{aligned}
$$

Not all of these coefficients are unconditionally positive: $a_{4}$ is only guaranteed positive for all $k_{\theta}^{\prime}$ and $k_{x}^{\prime}$ when $k\left(h+q_{1} d\right)>$ $q_{1} d$. Note that the translational rotor flapping coefficient, $q_{1}$, a fixed parameter of the helicopter — is a small value and this inequality will be satisfied for conventional systems.

The Routh-Hurwitz array analysis for this equation is more involved, owing to the system being fifth-order with three parameters: effective stiffnesses $k_{\theta}^{\prime}$ and $k_{x}^{\prime}$, and the gripper distance below the center of gravity, $d$. As the flight controller is considered fixed, suitable bounds must be found on these parameters to guarantee stability. However, as $d$ is a physical parameter of the helicopter and is not easily changed, it may be considered as effectively fixed (assuming $a_{4}>0$ is satisfied) and therefore only variation in linkage stiffness will be considered.

The Routh-Hurwitz array elements for a fifth-order polynomial are given by:

$$
\begin{aligned}
b_{1} & =\left(a_{1} a_{2}-a_{3}\right) / a_{1} \\
b_{2} & =\left(a_{1} a_{4}-a_{5}\right) / a_{1} \\
c_{1} & =\left(b_{1} a_{3}-a_{1} b_{2}\right) / b_{1} \\
c_{2} & =a_{5} \\
d_{1} & =\left(c_{1} b_{2}-b_{1} c_{2}\right) / c_{1} \\
e_{1} & =a_{5}
\end{aligned}
$$

The four leading entries of the array, $b_{1}, c_{1}, d_{1}$ and $e_{1}$, must all be positive for the system to be stable. With the exception of $c_{2}$ and $e_{1}$, the equations for the array elements are long and these are given in the appendix.

To understand what regions of stability exist, the value of each entry may be plotted as a function of $k_{\theta}^{\prime}$ and $k_{x}^{\prime}$ (see Fig. 68 ). The vertical scale of the plots are truncated to between 0 and 1000; shading represents magnitude (height) for clarity.

The system is stable where all three entries are greater than zero. These bounds appear as discontinuities in Fig. 7 and 8. These may be plotted a single superimposed graph showing the region of stable parameters (see Fig. 9).

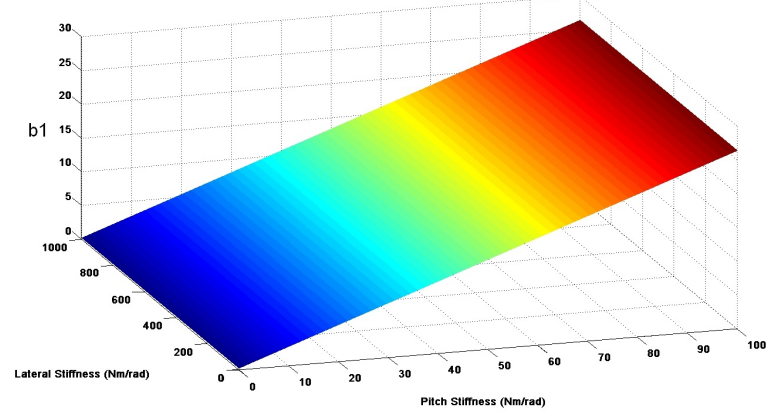

Figure 6. ROUTH-HURWITZ ELEMENT $b_{1}$.

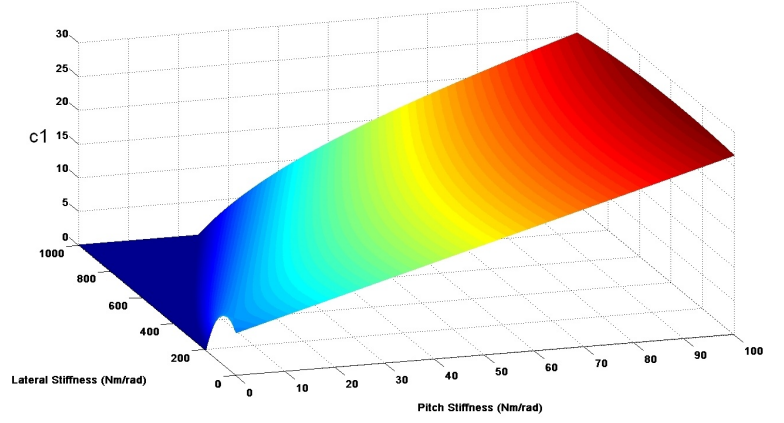

Figure 7. ROUTH-HURWITZ ELEMENT $c_{1}$.

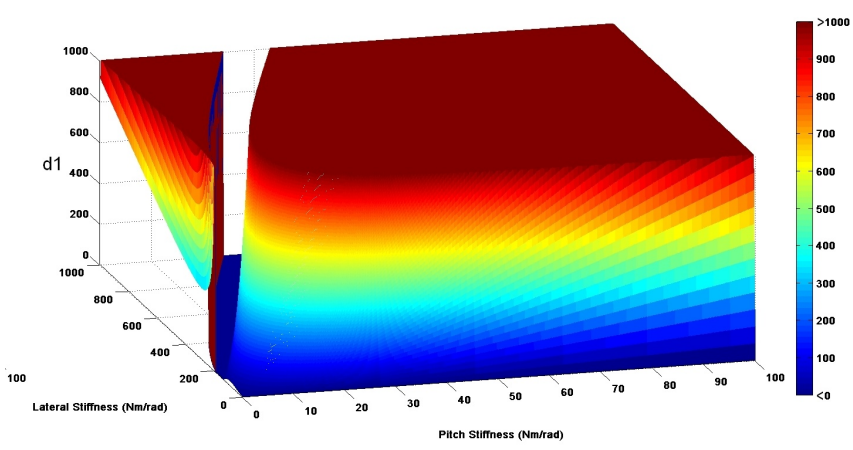

Figure 8. ROUTH-HURWITZ ELEMENT $d_{1}$.

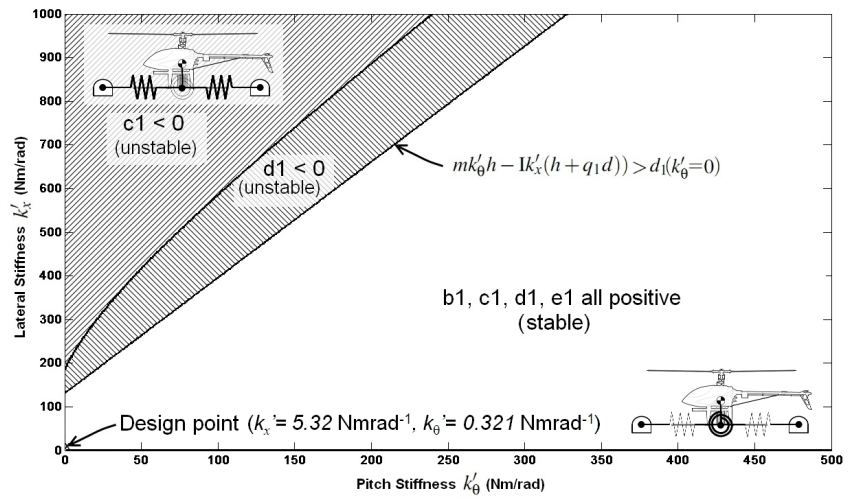

Figure 9. STABILITY BOUNDS FOR LINKAGE STIFFNESSES. 
Explicit bounds may be found by analyzing the equations for $c_{1}$ and $d_{1}$. Factorization of these entries is lengthy, but yields the following set of constraints for stability, given arbitrarily large values of $k_{\theta}^{\prime}$ and $k_{x}^{\prime}$ :

$$
\begin{aligned}
& 0<m k_{\theta}^{\prime}-\mathrm{I} k_{x}^{\prime} \\
& 0<m k_{\theta}^{\prime} k_{i}-\mathrm{I} k_{x}^{\prime} q_{1} d \\
& \left.0<m k_{\theta}^{\prime} h-\mathrm{I} k_{x}^{\prime}\left(h+q_{1} d\right)\right) \\
& 0<m^{2} g h^{2}-\mathrm{I} k_{x}^{\prime} q_{1} d
\end{aligned}
$$

The first two inequalities are derived from $c_{1}$, but are also required for $d_{1}$. The third and fourth are from $d_{1}$. The first three are constraints on the ratio of $k_{\theta}^{\prime}$ and $k_{x}^{\prime}$. The fourth is a constraint on the absolute magnitude of $k_{x}^{\prime}$. Of these, (42) is the most restrictive slope, although there is a region of stability under the intercept at $k_{\theta}^{\prime}=0$. This margin can be calculated explicitly by determining $d_{1}=0$ for $k_{x}^{\prime}=0$, which has the solution:

$$
k_{x 0}^{\prime}=\frac{g m^{2} h^{2}}{\mathrm{I}\left(h+q_{1} d\right)}
$$

Intuitively, the stable and unstable regimes can be understood at extrema as either a helicopter pinned to a rigid fixture but free to rotate $\left(k_{\theta}^{\prime}=0, k_{x}^{\prime}=\infty\right)$, or a helicopter fixed level but free to translate $\left(k_{\theta}^{\prime}=\infty, k_{x}^{\prime}=0\right)$ - analogous to an inverted pendulum.

\section{Proof-Of-Concept System Parameters And Stability}

The stability of the proof-of-concept system previously described can be determined using the helicopter, controller and gripper parameters given in Table 1. By applying (16) it can be readily verified that the PID parameters specified stabilize the helicopter in free flight.

Computing the tethered stability conditions, it is seen that the gripper offset requirement, $k\left(h+q_{1} d\right)>q_{1} d$, is satisfied, but the equivalent stiffnesses $k_{\theta}^{\prime}$ and $k_{x}^{\prime}$ produced by the proximal and distal joints, $k_{1}$ and $k_{2}$, and kinematic stiffness lifting full object mass, $m_{0}$, do not satisfy the strict slope requirement of (42) for guaranteed stability. It is therefore necessary to compute the intercept at $k_{\theta}^{\prime}=0$ to ascertain if the parameters are in the stable region. Using (44) and (42), the stability bound intercept is $132.6 \mathrm{Nmrad}^{-1}$, and the slope is $26.5 / 1$, placing the system well within stable operating conditions.

This provides good confidence that the helicopter-gripper system can be hovered in a grasping configuration without inducing instability. Although the experimental system has yet to be tested in this mode, trial results are expected shortly.

\section{CONCLUSION}

\begin{tabular}{|c|c|c|c|c|c|}
\hline \multicolumn{6}{|c|}{ Aircraft Parameters } \\
\hline$g$ & 9.81 & $\mathrm{~ms}^{-2}$ & $\rho$ & 1.184 & $\mathrm{kgm}^{-3}$ \\
\hline$m$ & 4.3 & $\mathrm{~kg}$ & I & 0.1909 & $\mathrm{kgm}^{2}$ \\
\hline$\omega$ & 96 & $\operatorname{rads}^{-1}$ & $R$ & 0.74 & $\mathrm{~m}$ \\
\hline$h$ & 0.1 & $\mathrm{~m}$ & $a$ & 5.5 & $\operatorname{rad}^{-1}$ \\
\hline$q_{1}$ & 0.0039 & & $q_{2}$ & 0.0266 & \\
\hline \multicolumn{6}{|c|}{ Control Parameters } \\
\hline$k$ & 0.2 & & $k_{d}$ & 2 & \\
\hline$k_{i}$ & 1 & & & & \\
\hline \multicolumn{6}{|c|}{ Gripper Parameters } \\
\hline$k_{1}$ & $2 \times 0.08$ & $\mathrm{Nmrad}^{-1}$ & $k_{\theta}$ & 0.16 & $\mathrm{Nmrad}^{-1}$ \\
\hline$k_{2}$ & $2 \times 0.4$ & $\mathrm{Nmrad}^{-1}$ & $k_{\theta}^{\prime}$ & 0.3207 & $\mathrm{Nmrad}^{-1}$ \\
\hline$k_{x}$ & 0.32 & $\mathrm{Nmrad}^{-1}$ & $k_{z}$ & 0.5867 & $\mathrm{Nm}^{-1}$ \\
\hline$k_{x}^{\prime}$ & 5.32 & $\mathrm{Nmrad}^{-1}$ & $k_{z}^{\prime}$ & 1.1733 & $\mathrm{Nm}^{-1}$ \\
\hline$l$ & 0.025 & $\mathrm{~m}$ & $d$ & 0.2 & $\mathrm{~m}$ \\
\hline$m_{0}$ & 1.0 & $\mathrm{~kg}$ & & & \\
\hline
\end{tabular}

By combining a simple planar model of a helicopter UAV in hover under PID control with a suspended bogie linkage representation of a compliant gripper, a dynamic model of an aerial
Table 1. AIRCRAFT, CONTROL AND GRIPPER PARAMETERS

manipulation system during object capture was developed. Using the Routh-Hurwitz criterion, a set of bounds on the gripper stiffness parameters was found to test the stability of a helicopterlinkage system. By applying these constraints to the known parameters of the aircraft, gripper and controller, it was shown that this particular configuration is well within the stable region of the constraints.

\section{Future Work}

Further analytical work will look more closely at the full non-linear, 6-DOF system and derive equivalent limits for system stability. Additions to the model will be made to consider the effect of pitching on lateral stiffness, and on the influence of stiffness induced by increased vertical thrust.

Once the conditions for guaranteed flight stability in full 3D flight are understood, the UAV will be used to attempt grasping an object while in hover. This will require modifications to the helicopter landing gear to raise the skids during flight and prevent unwanted ground contact.

\section{REFERENCES}

[1] Cai, G., Chen, B., and Lee, T., 2010. "An overview on development of miniature unmanned rotorcraft systems". Frontiers of Electrical and Electronic Engineering in China.

[2] Gentili, L., Naldi, R., and Marconi, L., 2008. "Modeling and control of VTOL UAVs interacting with the environment”. Proc. IEEE Conference on Decision and Control. 
[3] Amidi, O., Kanade, T., and Miller, J. R., 1998. "Visionbased autonomous helicopter research at Carnegie Mellon robotics institute 1991-1997". In American Helicopter Society International Conference.

[4] Borenstein, J., 1992. "The hoverbot - an electrically powered flying robot". ftp://ftp.eecs.umich.edu/people/johannb/paper99.pdf.

[5] Kuntz, N., and Oh, P., 2008. "Development of autonomous cargo transport for an unmanned aerial vehicle using visual servoing". Proc. Digital Systems and Control Conference.

[6] Dollar, A., and Howe, R., 2006. "A robust compliant grasper via shape deposition manufacturing". IEEE/ASME Transactions on Mechatronics, 11(2), pp. 154-161.

[7] Kaufman, L., and Schultz, E., 1962. "The stability and control of tethered helicopters". Journal of the American Helicopter Society, 7(4).

[8] Schmidt, G., and Swik, R., 1974. "Automatic hover control of an unmanned tethered rotorplatform". Automatica, 10(4), pp. 393 - 394, IN1-IN2, 395-403.

[9] Oh, S.-R., Agrawal, S., Pathak, K., Pota, H. R., and Garrett, M., 2005. "Autonomous helicopter landing on a moving platform using a tether". IEEE International Conference on Robotics and Automation.

[10] Oh, S.-R., Agrawal, S., Pathak, K., Pota, H. R., and Garrett, M., 2006. "Approaches for a tether-guided landing of an autonomous helicopter". IEEE Transactions on Robotics, 22, pp. 536-544.

[11] Mettler, B., 2002. Identification Modeling and Characteristics of Miniature Rotorcraft. Kluwer Academics Publisher, Nowell, MA.

[12] Pounds, P., Mahony, R., and Corke, P., 2010. "Modelling and control of a large quadrotor robot". Control Engineering Practice, 18(7), pp. 691 - 699. Special Issue on Aerial Robotics.

[13] Prouty, R., 2002. Helicopter Performance, Stability and Control, first ed. Krieger Publishing Company, London, United Kingdom.

[14] Franklin, G. F., Powell, J. D., and Emami-Naeini, A., 1994. Feedback Control of Dynamic Systems. Addison Wesley, Reading, MA.

[15] Bisgaard, M., Bendtsen, J., and la Cour-Harbo, A., 2009. "Modelling of generic slung load system". Journal of Guidance, Control and Dynamics, 32(2).

[16] Bernard, M., and Kondak, K., 2009. "Generic slung load transportation system using small size helicopters". Proc. International Conference on Robotics and Automation.

\section{Appendix: Routh-Hurwitz Array Elements}

The Routh-Hurwitz array is used in determining the stability of a characteristic polynomial [14]. It is computed from the polynomial coefficients $\left(a_{n}\right)$ and the determinant of preceding entries in the matrix. The entries of the Routh-Hurwitz array for (28) are given below. The elements have been factorized so that it can be seen by inspection how the conditions presented in (40)-(43) arise. The following substitutions are made for clarity:

$$
\begin{aligned}
& p_{1}=-\mathrm{I} q_{1} g-\mathrm{I} k k_{i}+\mathrm{I} q_{1} g k+m g h k\left(q_{2}+k k_{d}\right)>0 \\
& p_{2}=\left(\mathrm{I}-\left(q_{2}+k k_{d}\right) d m\right)
\end{aligned}
$$

$$
\begin{aligned}
& b_{1}= \frac{\left(p_{1} h m^{2}+k_{x}^{\prime} q_{1} \mathrm{I}^{2}+\left(q_{2}+k k_{d}\right) m\left(m h k_{\theta}^{\prime}-\mathrm{I} k_{x}^{\prime} q_{1} d\right)\right)}{\left(\mathrm{I} m\left(q_{2} h m+\mathrm{I} q_{1}+h k k_{d} m\right)\right)} \\
& b_{2}=\frac{\left(\mathrm{I} q_{1}+m g h q_{1} p_{2}+m\left(h+q_{1} d\right) p_{1}+\left(q_{2}+k k_{d}\right) h m k_{\theta}^{\prime}\right) k_{x}^{\prime}}{m \mathrm{I}\left(q_{2} h m+\mathrm{I} q_{1}+h k k_{d} m\right)} \\
& c_{1} \neq m q_{1} p_{1}+m^{3} h^{2}\left(k k_{i}+g q_{1}\right) p_{1}+m h q_{1} k k_{i} k_{x}^{\prime} \mathrm{I}^{2} \\
&+\left(q_{2}+k k_{d}\right)^{2} h q_{1} k_{x}^{\prime} d m\left(m k_{\theta}^{\prime}-\mathrm{I} k_{x}^{\prime}\right) \\
&+\left(q_{2}+k k_{d}\right)^{2} q_{1} k_{x}^{\prime} d m\left(m^{2} g h^{2}-\mathrm{I} k_{x}^{\prime} q_{1} d\right) \\
&+\left(q_{2}+k k_{d}\right) m^{2} h k k_{i}\left(h m k_{\theta}^{\prime}-\mathrm{I} k_{x}^{\prime} q_{1} d\right) \\
&+\left(q_{2}+k k_{d}\right) h q_{1}\left(m k_{\theta}^{\prime}-\mathrm{I} k_{x}^{\prime}\right)^{2} \\
&\left.+\left(q_{2}+k k_{d}\right) q_{1}\left(m k_{\theta}^{\prime}-\mathrm{I} k_{x}^{\prime}\right)\left(m^{2} g h^{2}-\mathrm{I} k_{x}^{\prime} q_{1} d\right)\right] \\
& /\left(p_{1} h m^{2}+\mathrm{I} q_{1} k_{x}^{\prime} \mathrm{I}+\left(q_{2}+k k_{d}\right) m\left(m h k_{\theta}^{\prime}-\mathrm{I} k_{x}^{\prime} q_{1} d\right)\right) \\
& c_{2}=\quad \frac{k k_{i} g m k_{x}^{\prime}\left(h+q_{1} d\right)}{\mathrm{I} m}
\end{aligned}
$$

$d_{1}=$

$$
\begin{aligned}
& k_{x}^{\prime}\left(\mathrm{I} q_{1}+h m\left(q_{2}+k k_{d}\right) k_{\theta}^{\prime}\right. \\
& \left.+m\left(h+q_{1} d\right) p_{1}+g m h q_{1} p_{2}\right)\left[m q _ { 1 } \left(h m k_{\theta}^{\prime}\right.\right. \\
& \left.-\mathrm{I} k_{x}^{\prime}\left(h+q_{1} d\right)\right) p_{1}+m^{3} h^{2}\left(k k_{i}+q_{1} g\right) p_{1}+\mathrm{I} m h q_{1} k k_{i} k_{x}^{\prime} p_{2} \\
& +\left(q_{2}+k k_{d}\right) m^{2} h^{2} k\left(m k_{\theta}^{\prime} k_{i}-\mathrm{I} k_{x}^{\prime} g q_{1}\right) \\
& +\left(q_{2}+k k_{d}\right) h q_{1}\left(m k_{\theta}^{\prime}-\mathrm{I} k_{x}^{\prime}\right)^{2} \\
& +\left(q_{2}+k k_{d}\right) h^{2} q_{1} m^{2} \mathrm{I} k_{x}^{\prime} k g \\
& +\left(q_{2}+k k_{d}\right)^{2} h q_{1} m k_{x}^{\prime} d\left(m k_{\theta}^{\prime}-\mathrm{I} k_{x}^{\prime}\right) \\
& +\left(q_{2}+k k_{d}\right) q_{1}\left(m k_{\theta}^{\prime}-\mathrm{I} k_{x}^{\prime}\right)\left(g m^{2} h^{2}-\mathrm{I} q_{1} k_{x}^{\prime} d\right) \\
& \left.+\left(q_{2}+k k_{d}\right)^{2} q_{1} k_{x}^{\prime} d m\left(g m^{2} h^{2}-\mathrm{I} q_{1} k_{x}^{\prime} d\right)\right] \\
& /\left[m \mathrm{I}\left(\left(q_{2}+k k_{d}\right) h m^{2} k_{\theta}^{\prime}+p_{1} h m^{2}+\mathrm{I} q_{1} k_{x}^{\prime} p_{2}\right)\right. \\
& \left.\quad *\left(q_{2} h m+\mathrm{I} q_{1}+h k k_{d} m\right)\right] \\
& -\frac{\left(p_{1} h m^{2}+\left(q_{2}+k k_{d}\right) h m^{2} k_{\theta}^{\prime}+\mathrm{I} q_{1} k_{x}^{\prime} p_{2}\right) k k_{i} g k_{x}^{\prime}\left(h+q_{1} d\right)}{\mathrm{I}^{2} m\left(q_{2} h m+\mathrm{I} q_{1}+h k k_{d} m\right)}
\end{aligned}
$$

$$
e_{1}=\quad \frac{k k_{i} g m k_{x}^{\prime}\left(h+q_{1} d\right)}{\mathrm{I} m}
$$

